\title{
The Effects of Offset Voltage on the Amplitude and Bandwidth of Kick-Out Pulses Used in the Nose-to-Nose Sampler Impulse Response Characterization Method
}

\author{
Donald R. Larson, Senior Member, IEEE, and Nicholas G. Paulter, Jr.
}

\begin{abstract}
The amplitude and bandwidth of kick-out pulses used in the nose-to-nose sampler impulse response characterization method were measured as a function of offset voltage. The measuring instrument was of similar bandwidth and its effects on the measurement were not removed. The amplitude is almost linear for offset voltages from $-500 \mathrm{mV}$ to $500 \mathrm{mV}$, except for values from about $-50 \mathrm{mV}$ to $50 \mathrm{mV}$. Slight changes in bandwidth were observed for offset voltages from $-250 \mathrm{mV}$ to $250 \mathrm{mV}$ with significant bandwidth loss observed for offset voltages outside this range.
\end{abstract}

Index Terms-High-speed samplers, impulse response, sampling oscilloscopes, waveform spectrum.

\section{INTRODUCTION}

C HARACTERIZATION of the impulse response or transfer function of high-speed/high-bandwidth samplers used in digital sampling oscilloscopes can be performed with either time or frequency-domain techniques. Because of the difficulty in obtaining phase information using frequency-domain methods, however, time-domain techniques are typically used. These time-domain methods require having pulse generators that have significantly greater bandwidth than the samplers themselves or, if the generators have comparable bandwidth, then their pulse profiles must be accurately known so that their influence can be deconvolved. Some time-domain methods that are being explored for sampler impulse response characterization use a photoconductively-generated electrical pulse for the reference pulse, and this pulse is measured using photoconductive or electro-optical sampling [1], [2]. Consequently, these methods may require that the electrical impulse response of the sampling photoconductor or electro-optic element also be known or measurable. This requirement, of course, brings us to the original problem of delivering a known input pulse to the samplers from which a sampler impulse response can be derived. The nose-to-nose sampler impulse response characterization method, introduced several years ago, is a method where a sampler is used as a pulse generator. The sampler generates a pulse (the kick-out pulse) when it is operated at a nonzero offset voltage, and it is argued that the

Manuscript received May 4, 2000; revised February 27, 2001.

The authors are with the National Institute of Standards and Technology, Gaithersburg, MD 20899-8111 (e-mail: donald.larson@nist.gov; nicholas.paulter@nist.gov).

Publisher Item Identifier S 0018-9456(01)06014-4. kick-out pulse represents the impulse response of the sampler [3]-[5]. This kick-out phenomena is limited to a particular sampler architecture.

Since it would be difficult to determine the kick-out pulse of a sampler using only that sampler, a series of kick-out pulses are measured using a set of three samplers. Each sampler in turn acts as a source of the kick-out pulses that are measured using the other samplers. The assumption that the sampler impulse response is very similar to the kick-out pulse cannot be valid unless the frequency or time response of the kick-out pulse is independent of the offset voltage. Furthermore, the utility of using a kick-out-pulse-derived sampler impulse response for purposes of calibration is dependent on the sampler's response being linear over its signal range.

\section{EXPERIMENT}

The nose-to-nose method gets its name from the arrangement of the samplers in the measurement procedure; that is, the input connectors of two different sampling heads are connected together using a male-male coaxial adapter. The sampling heads are physically and electrically situated such that the distance between their input connectors is minimized, resulting in a nose-to-nose appearance. Two $50 \mathrm{GHz}$ digital sampling oscilloscopes were used and, in our implementation, both sampling heads are triggered using a short transition duration step (17 ps transition duration, $0.25 \mathrm{~V}$ amplitude before a wideband splitter) with a $2 \mathrm{kHz}$ repetition rate. The kick-out pulse also contains a signal contribution from the strobe pulse that couples into the sampling diodes. To minimize the strobe-coupling effect, the following procedure is used [3]. Two kick-out waveforms are acquired, one using a negative offset voltage [Fig. 1(a)] and another using a positive offset voltage of equal magnitude [Fig. 1(b)]. The polarity of the kick-out pulse changes sign with the offset voltage but the strobe pulse polarity does not. Subtracting these two kick-out pulse waveforms will produce a difference waveform in which the strobe pulse contribution has been minimized and the kick-out pulse amplitude has been doubled. Dividing this difference waveform by two yields an estimate of the kick-out pulse with the strobe pulse contribution removed [Fig. 1(c)]. The offset voltages for the sampling head used to generate the kick-out pulse are $+V_{i}$ (for the positive offset voltage) and $-V_{i}$ (for the negative offset voltage), and the offset voltage of the sampling head 


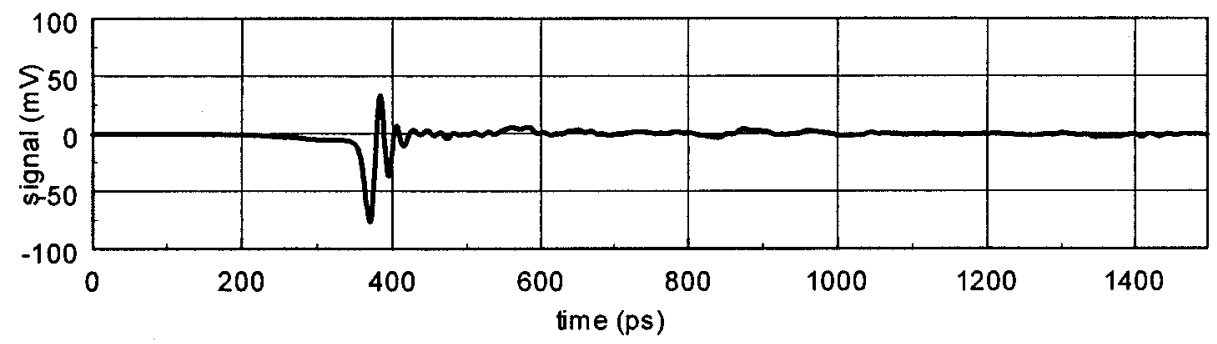

(a)

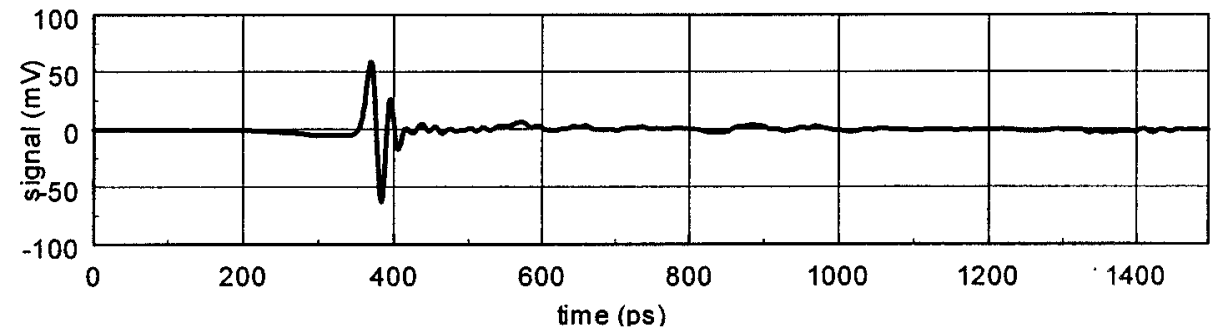

(b)

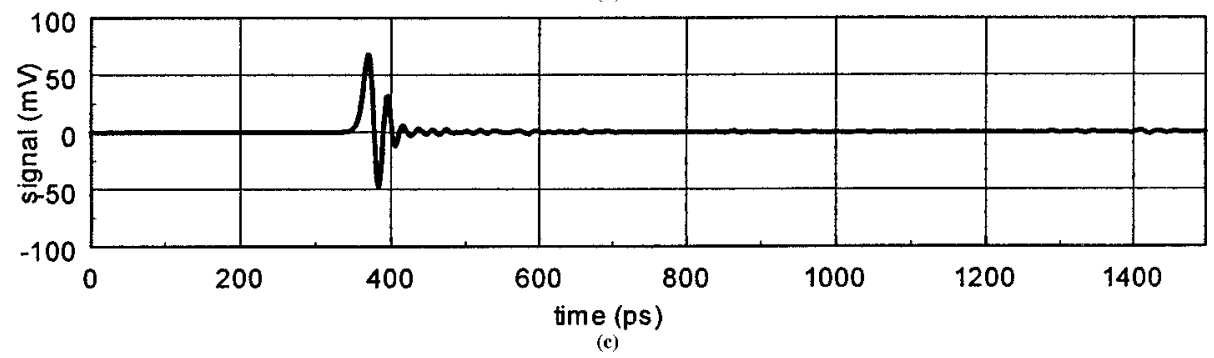

Fig. 1. Kick-out pulses acquired using (a) negative $200 \mathrm{mV}$ offset voltage, (b) positive $200 \mathrm{mV}$ offset voltage, and (c) the difference waveform (divided by two).

measuring the kick-out pulse is set to $0.0 \mathrm{~V}$. The offset voltage is the voltage generated internally by the oscilloscope with the offset voltage setting of the vertical axis of the oscilloscope. We chose a $1.5 \mathrm{~ns}$ epoch with a 1024 element waveform. The pulse duration of the difference waveform is approximately 11 ps (full duration at half maximum); however, the waveform exhibits significant aberrations.

Kick-out waveforms were acquired with offset voltages ranging from $-500 \mathrm{mV}$ to $+500 \mathrm{mV}$ (the limits of the oscilloscope) in $25 \mathrm{mV}$ increments. A waveform acquired with a negative offset voltage will be referred to as $W_{-V}(t)$ and when acquired with a positive offset voltage, $W_{+V}(t)$. The oscilloscope was operated in averaging mode and each waveform acquired from the oscilloscope is the result of 256 internally averaged waveforms (by the oscilloscope). The acquired waveforms were transferred to a personal computer for processing. These acquired waveforms, $W_{-V}(t)$ and $W_{+V}(t)$, are the result of the convolution of the kick-out pulse, the impulse response of the male-male adapter, and the impulse response of the measuring sampler. Difference waveforms, $D_{\mathrm{V}}(\mathrm{t})=\left(\mathrm{W}_{-\mathrm{V}}(\mathrm{t})-\mathrm{W}_{+\mathrm{V}}(\mathrm{t})\right) / 2$, were calculated to get estimates of the kick-out pulse amplitude and its transfer function magnitude without strobe pulse contributions.

\section{RESULTS}

The peak-to-peak (p-p) amplitude of the kick-out pulses $\left[W_{-V}(t)\right.$ and $\left.W_{+V}(t)\right]$ is plotted as a function of offset voltage in Fig. 2. The $\mathrm{p}-\mathrm{p}$ amplitude was obtained by subtracting the minimum waveform voltage from the maximum waveform voltage. The $\mathrm{p}-\mathrm{p}$ amplitude is used because both positive and negative pulses are being considered and because of the large aberrations exhibited in the acquired waveforms. Results similar to those shown in Fig. 2 were also obtained when just the maximum waveform voltage values were used. The graph is asymmetric with offset voltage because the strobe pulse reduces the kick-out pulse amplitude for negative offset voltages and increases the kick-out pulse amplitude for positive offset voltages.

The amplitude of $\mathrm{D}_{\mathrm{V}}(\mathrm{t})$ is nearly linear for offset voltages in the range of $50 \mathrm{mV}$ to $500 \mathrm{mV}$. This is depicted in Fig. 3. The random (Type A) component of uncertainty in the amplitude of $\mathrm{D}_{\mathrm{V}}(\mathrm{t})$ is $0.9 \mathrm{mV}$, as determined from a series of measurements on the difference waveforms (all measurement uncertainties presented herein represent one standard deviation).

The Fourier transforms of the difference waveforms were calculated and a typical result, normalized to the dc value of the spectrum, is depicted in Fig. 4. The frequency at which the magnitude of the spectrum of $D_{\mathrm{V}}(t)$ drops to $-3 \mathrm{~dB}$ of its value at $\mathrm{dc}$ is plotted as a function of offset voltage in Fig. 5. The results show a slight decrease in bandwidth with increasing offset voltage up to around $\pm 250 \mathrm{mV}$. For offset voltages outside this range, the decrease becomes more significant. The $-3 \mathrm{~dB}$ frequency drops from approximately $50.3 \mathrm{GHz}$ for a $\pm 200 \mathrm{mV}$ offset voltage to approximately $45.8 \mathrm{GHz}$ for a $\pm 500 \mathrm{mV}$ offset voltage (Fig. 5). The maximum random (Type A) component 


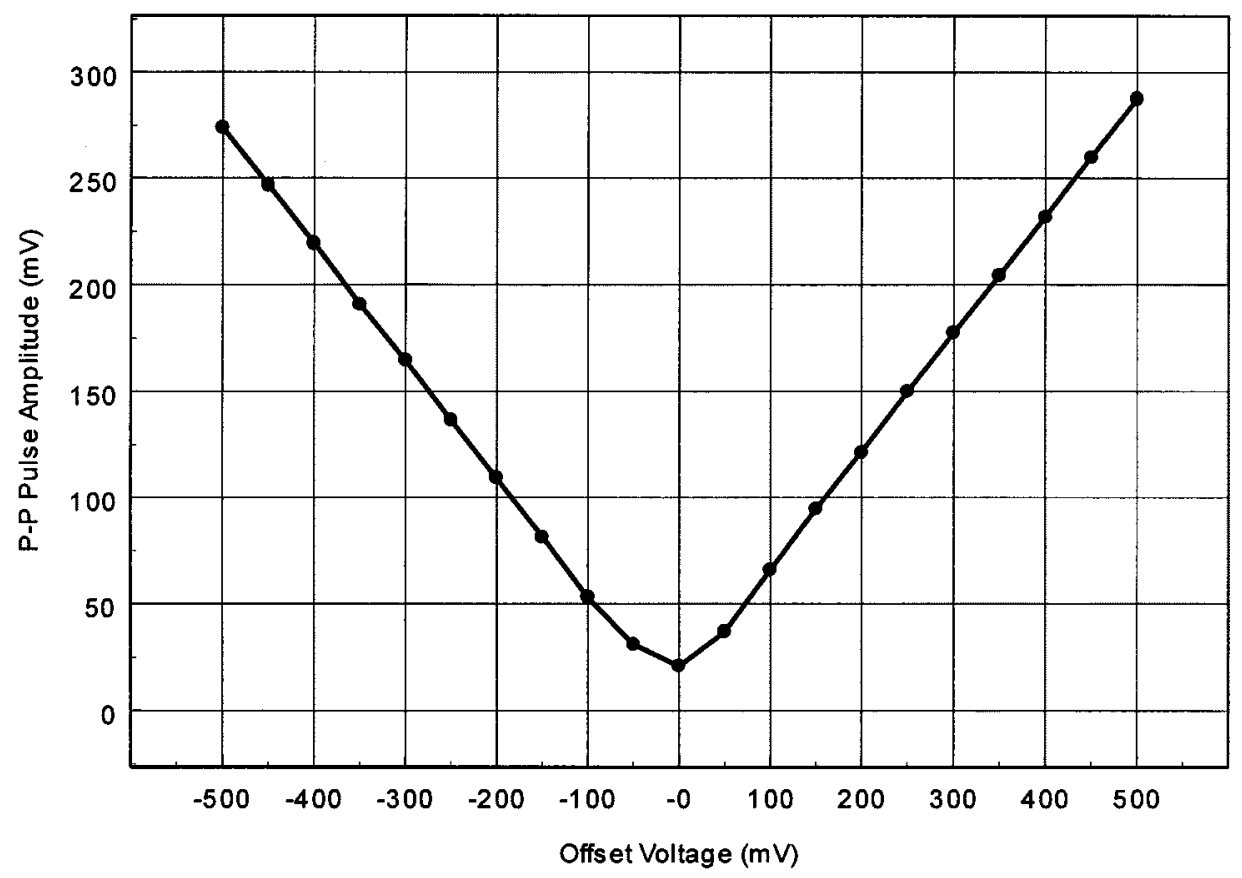

Fig. 2. Peak-to-peak amplitude of acquired kick-out pulses as a function of offset voltage.

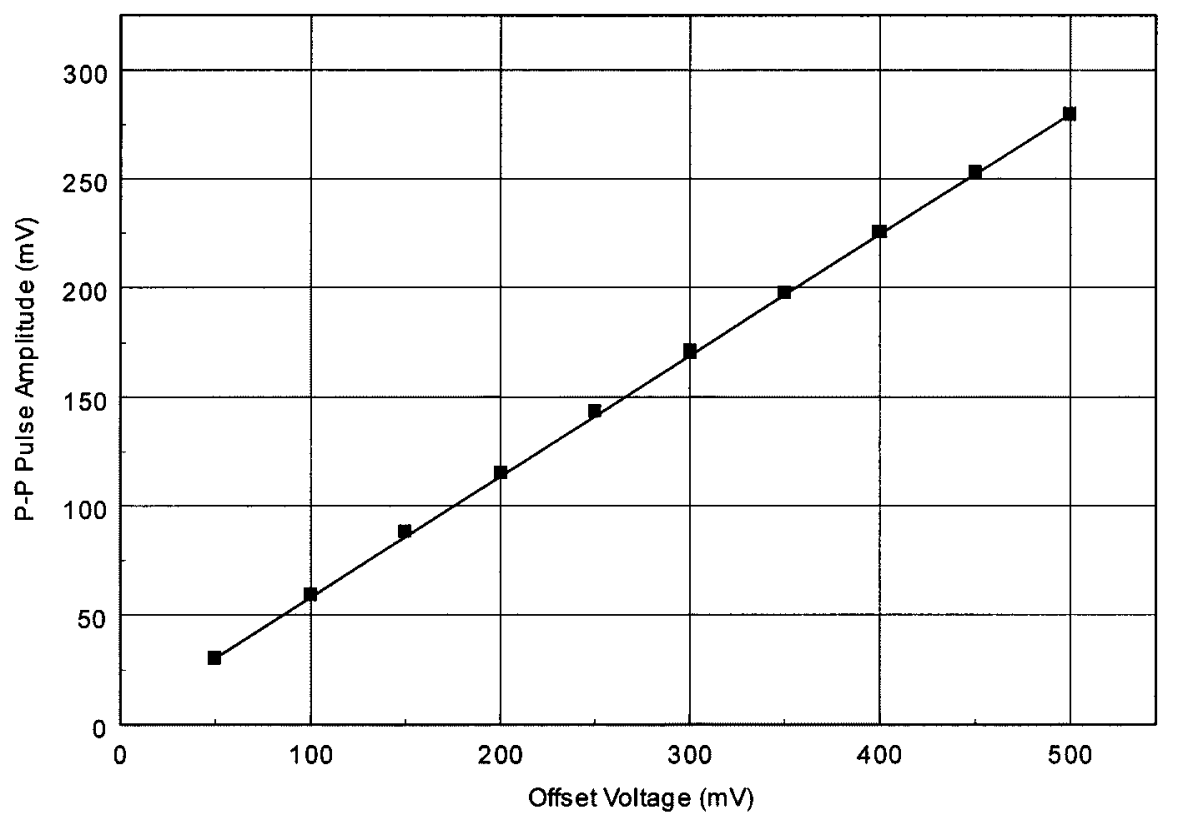

Fig. 3. Peak-to-peak amplitude of the difference waveforms as a function of offset voltage (shown with a line having endpoints at $50 \mathrm{mV}$ and $500 \mathrm{mV}$ ).

of uncertainty for all frequency components of the spectrum of $\mathrm{D}_{\mathrm{V}}(\mathrm{t})$ is $0.19 \mathrm{~dB}$. It should be noted that $\mathrm{D}_{\mathrm{V}}(\mathrm{t})$ is the convolution of the kick-out pulse with the sampler impulse response and the response of the coaxial adapter.

The magnitude of the spectrum of $D_{\mathrm{V}}(\mathrm{t})$ was also examined as a function of the number of averages. The variation in magnitude is less than or equal to the maximum random uncertainty noted above when the number of averages is in the range of 32 to 2048. This implies that during the time required to acquire the waveforms for averaging, the pulse distortion from signal drift and time jitter does not increase significantly. For waveforms acquired using less than 32 averages, the variation in spectral magnitude increased due to signal noise.

\section{CONCLUSIONS}

The variation in the $-3 \mathrm{~dB}$ bandwidth observed indicates that the range of offset voltages used in the nose-to-nose method must be limited to less than $250 \mathrm{mV}$ for most pulse metrology applications. The variation in bandwidth is minimal (the same as the statistical variation) for offset voltage magnitudes greater than $50 \mathrm{mV}$ and less than $250 \mathrm{mV}$ (Fig. 5); thus, only offset 


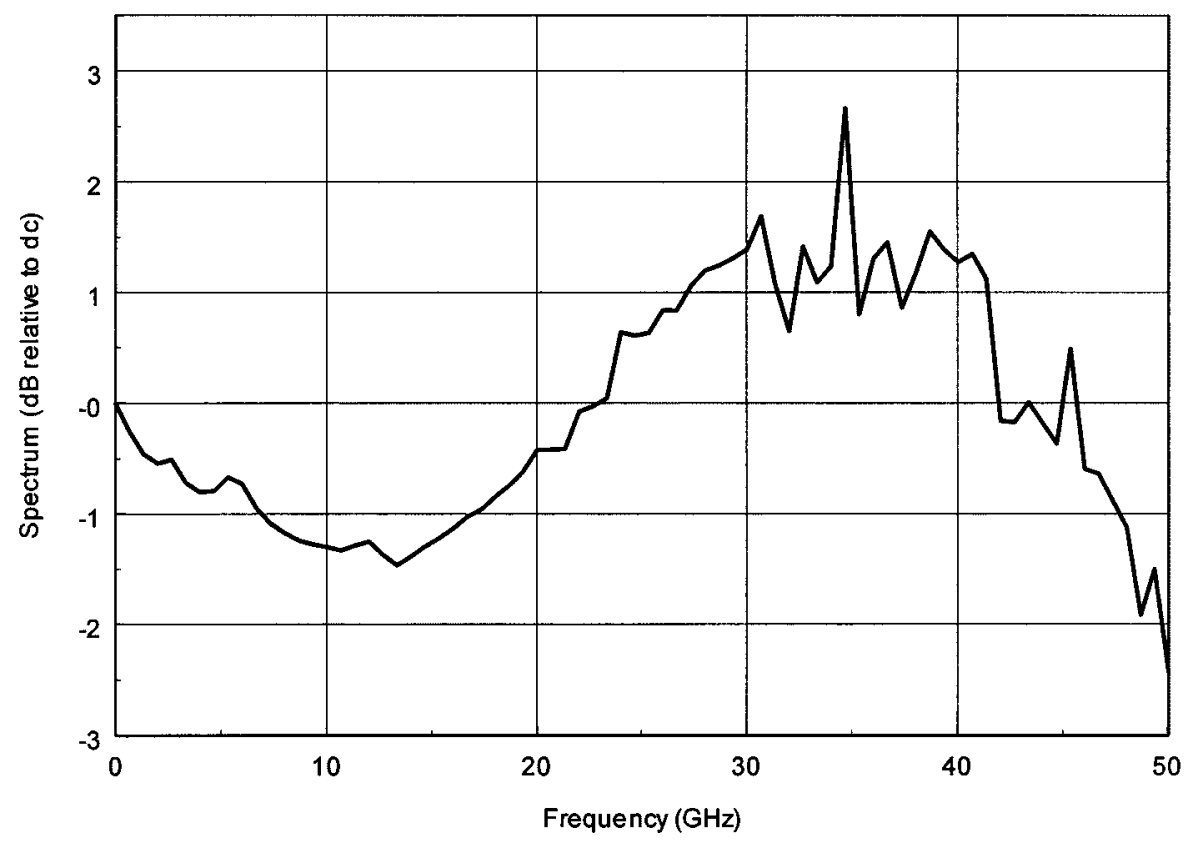

Fig. 4. Typical difference waveform spectrum (normalized to the dc value of the spectrum).

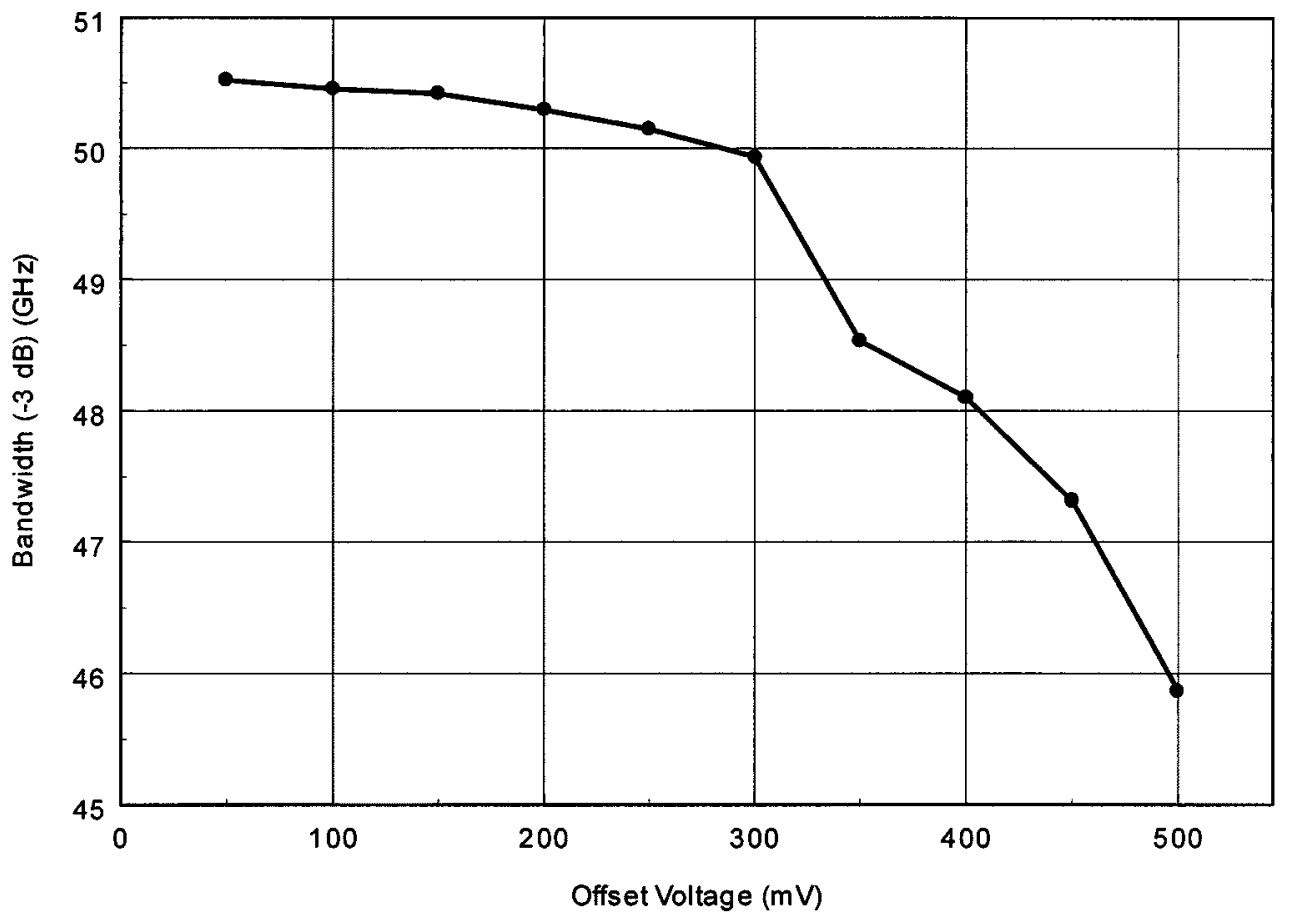

Fig. 5. The $-3 \mathrm{~dB}$ bandwidth of difference waveform spectra as a function of offset voltage.

voltages in this range should be used. This magnitude of offset voltage limits the usable kick-out pulse amplitude to less than $125 \mathrm{mV}$, which is less than the input amplitude range of the oscilloscope $(\approx 800 \mathrm{mV})$. The decrease in bandwidth with increasing offset voltage is probably due to saturation of the kick-out pulse generation mechanism and is not a limitation in the sampling mechanism; however, we have not confirmed this. The effect of the kick-out pulse and strobe pulse on devices connected to the sampler input also needs to be investigated. At least 32 averages should be used to acquire the kick-out pulse waveforms to minimize the effect of noise on these waveforms.

\section{ACKNOWLEDGMENT}

The authors would like to thank T. M. Souders and P. D. Hale of the National Institute of Standards and Technology for their 
useful discussions. They would also like to thank B. A. Bell of the National Institute of Standards and Technology for administrative support.

\section{REFERENCES}

[1] D. Henderson and A. G. Roddie, "Calibration of fast sampling oscilloscopes," Meas. Sci. Technol., vol. 1, pp. 673-679, 1990.

[2] A. J. A. Smith, A. G. Roddie, and D. Henderson, "Electrooptic sampling of low temperature GaAs pulse generators for oscilloscope calibration," Opt. Quant. Electron., vol. 28, pp. 933-943, 1996.

[3] K. Rush, S. Draving, and J. Kerley, "Characterizing high-speed oscilloscopes," IEEE Spectrum, pp. 38-39, Sept. 1990.

[4] J. Verspecht and K. Rush, "Individual characterization of broadband sampling oscilloscopes with a nose-to-nose calibration procedure," IEEE Trans. Instrum. Meas., vol. 43, pp. 347-354, Apr. 1994.

[5] J. Verspecht, "Broadband sampling oscilloscope characterization with the 'nose-to-nose' calibration procedure: A theoretical and practical analysis," IEEE Trans. Instrum. Meas., vol. 44, pp. 991-997, Dec. 1995.

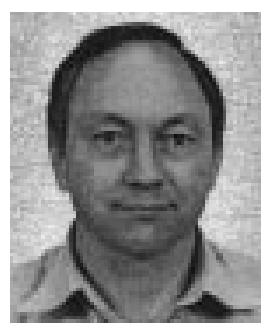

Donald R. Larson (M'79-SM'94) received the B.S. degree (cum laude) from Brigham Young University, Provo, Utah, in 1978 and the M.S.E.E. degree from the University of Colorado, Boulder, in 1981.

$\mathrm{He}$ worked at the National Institute of Standards and Technology (NIST) in Boulder from 1976 until 1998 in the Optoelectronics Division. Since 1998, he has been at NIST, Gaithersburg, MD, in the Electricity Division.

Nicholas G. Paulter Jr. received the M.S. degree in chemistry from the University of New Mexico, Albuquerque, in 1988 and the M.S. degree in electrical engineering from the University of Colorado, Boulder, in 1990.

He was with Los Alamos National Laboratory, Los Alamos, NM, from 1980 to 1989 , and was involved in the study of fast electrical phenomena and in the development of high-speed photoconductors for use as ultrafast light detectors and sampling gates. In 1989, he joined the National Institute of Standards and Technology (NIST), Boulder, to develop transient pulse measurement techniques and analysis. He is presently with NIST, Gaithersburg, MD. His present research interests include semiconductor physics, materials properties, electrooptics, ultrafast electronic phenomena, and waveform/data processing and analysis. 